\title{
Inactivation of the entire Arabidopsis group II GH3s confers tolerance to salinity and drought
}

Rubén Casanova-Sáez ${ }^{1,2 *}$, Eduardo Mateo-Bonmatí1,2,3 ${ }^{1,}$ Jan Šimura $^{1}$, Aleš Pěnčík ${ }^{4}$, Ondřej Novák ${ }^{1,4}$, Paul Staswick ${ }^{5}$, and Karin Ljung ${ }^{1 *}$

${ }^{1}$ Umeå Plant Science Centre (UPSC), Department of Forest Genetics and Plant Physiology, Swedish University of Agricultural Sciences, 90183 Umeå, Sweden

${ }^{4}$ Laboratory of Growth Regulators, Faculty of Science, Palacký University and Institute of Experimental Botany of the Czech Academy of Sciences, Šlechtitelů 27, Olomouc, Czech Republic

${ }^{5}$ Department of Agronomy and Horticulture, University of Nebraska, Lincoln, NE, USA

*Corresponding authors: Rubén Casanova-Sáez (ruben.casanova.saez@slu.se) and Karin Ljung (karin.ljung@slu.se)

${ }^{2}$ These authors contributed equally to this work.

${ }^{3}$ Current address: John Innes Centre, NR4 7UA, Norwich, United Kingdom

Running head: Blocking IAA GH3s confers tolerance to water deprivation

Keywords: Arabidopsis, auxin, GH3, drought, salinity, stress tolerance

ORCID (R.C.-S.): 0000-0001-5683-7051

Twitter (R.C.-S.): @rcasanovasaez

ORCID (E.M.-B.): 0000-0002-2364-5173

Twitter (E.M.-B.): @emateobonmati

ORCID (J.Š.): 0000-0002-1567-2278

ORCID (A.P.): 0000-0002-1314-2249

ORCID (O.N.): 0000-0003-3452-0154

ORCID (P.S.): 0000-0003-2798-0275

ORCID (K.L.): 0000-0003-2901-189X

Twitter (K.L.): @GroupLjung 


\section{ABSTRACT}

36 Indole-3-acetic acid (IAA) controls a plethora of developmental processes. Thus, regulation of

37 their levels is of great relevance for plant performance. Cellular IAA concentration depends on

38 the combined result of its transport, biosynthesis and various redundant pathways to inactivate

39 IAA, including oxidation and conjugation. Group II members of the GRETCHEN HAGEN 3

40 (GH3) gene family code for acyl acid amido synthetases catalysing the conjugation of IAA to

41 amino acids. However, the high level of functional redundancy among them has hampered

42 thorough analysis of their roles in plant development. In this work, we generated an

43 Arabidopsis gh3.1,2,3,4,5,6,9,17 (gh3oct) mutant to knock-out the group II GH3 pathway. The

44 gh3oct plants had an improved root architecture, were more tolerant to osmotic stresses due

45 to locally increased IAA levels and were more drought tolerant. IAA metabolite quantification

46 in gh3oct plants suggested the existence of additional GH3-like enzymes in IAA metabolism.

47 Moreover, our data suggested that oxIAA production depends, at least partly, on the GH3

48 pathway. Targeted stress-hormone analysis further suggested an involvement of ABA in the

49 differential response to salinity of gh3oct plants. Taken together, our data provide new insights

50 into the roles of group II GH3s in IAA metabolism and hormone-regulated plant development. 


\section{INTRODUCTION}

Regulation of the levels and distribution of the major auxin indole-3-acetic acid (IAA) is of pivotal importance for plant development because it regulates multiple processes, including embryo, seed, root and leaf development, meristem maintenance, shoot branching and responses to environmental stresses (Casanova-Saez \& Voss, 2019; Leftley et al., 2021). Spatiotemporal fluctuations of IAA levels in response to external and internal stimuli drive plant developmental responses by triggering signalling cascades that result in modulation of cell division, differentiation and growth (Gallei et al., 2020).

Control of IAA levels and distribution within plant cells and tissues is achieved by intercellular and intracellular transport mechanisms (Band, 2021; Hammes et al., 2021) and spatiotemporally regulated IAA biosynthesis and inactivation (Zhao, 2018; Casanova-Saez et al., 2021). IAA metabolic inactivation primarily involves oxidation processes and the formation of IAA-sugar and IAA-amino acid (IAA-aa) conjugates (Casanova-Saez et al., 2021). IAA oxidation is facilitated by the angiosperm-related DIOXYGENASE FOR AUXIN OXIDATION (DAO) proteins (Zhao et al., 2013; Porco et al., 2016; Zhang et al., 2016; Takehara et al., 2020). The formation of oxIAA is an irreversible IAA inactivation process that operates to remove excess auxin, as inferred from higher oxIAA formation detected at sites of IAA maxima (Pencik et al., 2013). Formation of ester-linked IAA conjugates with glucose (IAA-glc) is a reversible reaction known to regulate IAA levels and homeostasis. Several members of the UDP-glucosyltransferase superfamily have been shown to mediate the conjugation of both IAA and oxIAA to glucose in vivo (Mateo-Bonmati et al., 2021).

IAA is also inactivated by conjugation to amino acids via amide bonds, a reaction catalysed by members of the GRETCHEN HAGEN 3 (GH3) family of acyl acid amido synthetases (Staswick et al., 2005). Most of these conjugates, including IAA-leucine, IAA-alanine and IAAphenylalanine, can be hydrolysed to free IAA, and therefore serve as IAA storage forms (LeClere et al., 2002; Rampey et al., 2004). However, IAA irreversibly conjugates to aspartate (IAA-Asp) and glutamate (IAA-Glu) during catabolism (Ostin et al., 1998; Rampey et al., 2004). IAA-Asp and IAA-Glu catabolites have been found at higher levels compared with reversible IAA-aa conjugates in different plant species (Kowalczyk \& Sandberg, 2001; Kojima et al., 2009; Pencik et al., 2009). Several IAA-Asp metabolites, including 6-OH-IAA-Asp and oxIAA-Asp, have been identified in the last few decades (Ostin et al., 1998; Kai et al., 2007). Recently, the IAA oxidase DAO1 was found to additionally operate in the GH3 pathway by mediating the formation of oxIAA-Asp from IAA-Asp in tobacco and Arabidopsis (Müller et al., 2021).

Based on sequence homology and substrate specificity studies, 19 Arabidopsis GH3 
proteins have been classified into three functionally diverse groups, of which group II consists of eight GH3 members with the ability to mediate the conjugation of IAA to amino acids, i.e. GH3.1, GH3.2, GH3.3, GH3.4, GH3.5, GH3.6, GH3.9 and GH3.17 (Staswick et al., 2002; Staswick et al., 2005). These group II GH3s are conserved across land plants (Terol et al., 2006).

Even though all Arabidopsis group II GH3s can conjugate IAA to different amino acids, substrate preferences exist among them. For instance, GH3.17 preferentially conjugates IAA to Glu, GH3.3 and GH3.4 preferentially conjugate IAA to Asp, and GH3.2, GH3.5 and GH3.6 show a similar predilection for Asp and Glu (Staswick et al., 2005; Brunoni et al., 2019). A range of amino acid substrate specificities has also been documented for GH3 orthologs in other plant species, e.g. Physcomitrella patens (Ludwig-Muller et al., 2009), Vitis vinifera (Bottcher et al., 2011) and Picea abies (Brunoni et al., 2020). Group II GH3s are also known to be promiscuous regarding the acyl acid substrate. GH3.3, GH3.5 and GH3.6 have been found to modulate jasmonic acid homeostasis by mediating its conjugation to amino acids (Gutierrez et al., 2012). GH3.5 has been further shown to mediate the conjugation of the auxin phenylacetic acid, and the benzoates salicylic acid and benzoic acid (Westfall et al., 2016).

Although specific roles have been reported for individual group II GH3 members (Khan \& Stone, 2007; Park et al., 2007; Du et al., 2012; Zheng et al., 2016; Di Mambro et al., 2017; Kirungu et al., 2019), their overlapping expression domains and functions in IAA inactivation have likely masked additional roles of these GH3s in IAA metabolism and plant development. In the present study, we generated a group II GH3 octuple knockout to bypass the redundancy in GH3-mediated IAA metabolism. The mutant plants showed pleiotropic high-auxin-related phenotypes, such as longer hypocotyls and petioles, as well as a more branched root system with no apparent penalty on primary root growth. Phenotypic, physiological and targetedhormonomic analyses further indicated that group II GH3s redundantly modulate IAA-dependent salinity tolerance and that they collectively participate in the response to drought. We also obtained evidence supporting a connection between the GH3 pathway and oxIAA production. Finally, our data suggested that additional $\mathrm{GH} 3$ or $\mathrm{GH} 3$-like genes might operate in IAA conjugation to amino acids.

\section{MATERIALS AND METHODS}

\section{Plant material and culture conditions}

Seeds from the Arabidopsis wild-type accession Col-0 and Ler and from the gh3 insertional lines (Table S1) were obtained from the Nottingham Arabidopsis Stock Centre 
119 (http://arabidopsis.info) and from the Arabidopsis Biological Resource Center 120 (https://abrc.osu.edu). The presence and position of all insertions were confirmed by PCR 121 amplification using gene-specific primers together with the insertion-specific primers Ds5'-1 122 (for SGT lines), 3'-dSpm (for SM lines) and LBb1.3 (for SALK lines) (see Tables S1 and S2).

Arabidopsis seeds were surface sterilized with a bleach solution $(40 \% \mathrm{vol} / \mathrm{vol}$ commercial bleach in $\mathrm{dH}_{2} \mathrm{O}$ and $0.002 \%$ Triton $\mathrm{X}-100$ ) for 8 min and then washed four times with sterile deionized water. Seeds were stratified for a minimum of 2 days and then sown under sterile conditions on square petri dishes containing half-strength Murashige \& Skoog salt mixture

127 (Duchefa, M0221), 1\% sucrose, 0.05\% MES hydrate (Sigma, M2933) and 0.8\% plant agar 128 (Duchefa, P1001) with the $\mathrm{pH}$ adjusted to 5.7 witgh potassium hydroxide. Flowering plants were grown in pots containing a 3:1 mixture of organic soil and vermiculite. All plants were grown, unless otherwise stated, under long-day conditions (16 h light, $8 \mathrm{~h}$ darkness) at $22 \pm$ $1^{\circ} \mathrm{C}$ under cool white fluorescent light $\left(150 \mu \mathrm{mol}\right.$ photons $\left.\mathrm{m}^{-2} \mathrm{~s}^{-1}\right)$. Flowering time was recorded as the number of rosette leaves at bolting under long- and short-day ( $8 \mathrm{~h}$ light, $16 \mathrm{~h}$ darkness) conditions.

\section{Chemical treatments and drought experiments}

Salinity and osmotic treatments were performed on half-strength MS plates supplemented with sodium chloride, D-sorbitol or D-mannitol before autoclaving. The inhibitors of YUCCAmediated IAA biosynthesis yucasin [5-(4-chlorophenyl)-4H-1,2,4-triazole-3-thiol] and PPBo (4-phenoxyphenylboronic acid), kindly provided by Prof. Ken-ichiro Hayashi, were prepared in DMSO stock solutions. IAA treatments were performed on MS plates supplemented with indole-3-acetic acid from a stock solution in absolute ethanol. Yucasin, PPBo and IAA were added to the autoclaved MS media before pouring into plates. To mimic drought conditions in vitro, we prepared half-strength MS plates with a reduced water potential as described previously (Verslues et al., 2006). Solidified MS medium was perfused with an overlay solution containing poly(ethylene glycol) (PEG8000; Sigma, P2139). Control plates were perfused with an overlay MS solution lacking PEG8000. For drought treatment on soil-grown plants, stratified seeds were planted in pots containing $40 \mathrm{~g}$ of soil mixture. Initially, 1 litre of water was added to each tray. Irrigation was then withheld until both gh3oct and wild-type plants displayed dryness symptoms. The weight of the pots was recorded to estimate and compare the water loss in the pots for each genotype. 
Around $14 \mu \mathrm{g}$ of nuclear-enriched DNA was purified from $1 \mathrm{~g}$ of gh3oct seedlings using a previously described protocol (Hanania et al., 2004). Whole-genome sequencing of the sample was performed at BGI Hong Kong using a BGISEQ-500 sequencing platform. 47 million 150bp-long reads were obtained, reaching a 59X genome depth. Trimmed fastq files were used to map the different insertions using Easymap software (Lup et al., 2021). Raw reads were deposited in the Short Read Archive (SRA) with the code SRX8771538.

\section{Gene expression analyses}

For expression analyses, RNA was isolated using the Total RNA Purification Kit (Norgen). DNA was removed using the RNase-Free DNase I Kit (Norgen). First-strand cDNA synthesis was performed with the iScript cDNA Synthesis Kit (BioRad). ACTIN2 gene was used as an internal control for relative expression quantification. Four biological replicates (each being a pool of several plants) were analysed in triplicate. qPCR reactions were performed in $10 \mu \mathrm{l}$ reactions containing $4 \mu$ of LightCycler 480 SYBR Green I Master (Roche), $4 \mu$ l of PCR-grade water (Roche), $1 \mu \mathrm{l}$ of the corresponding primer pair (10 $\mu \mathrm{M}$ each) and $1 \mu \mathrm{l}$ of the cDNA template. The primers used are listed in Table S2. Quantification of relative gene expression was performed using the comparative $\mathrm{C}_{\mathrm{T}}$ method $\left(2-{ }^{\Delta \Delta C^{t}}\right)$ (Schmittgen \& Livak, 2008) on a CFX384 Touch Real-Time PCR Detection System (BioRad).

\section{Quantification of IAA and IAA metabolites}

Extraction and purification of the targeted compounds (IAA, oxIAA, IAA-Asp, IAA-Glu, IAAglc, oxIAA-glc) were performed according to (Novak et al., 2012). Briefly, $10 \mathrm{mg}$ of frozen material per sample was homogenized using a bead mill $\left(27 \mathrm{~Hz}, 10 \mathrm{~min}, 4^{\circ} \mathrm{C}\right.$; MixerMill, Retsch $\mathrm{GmbH}$, Haan, Germany) and extracted in $1 \mathrm{ml}$ of $50 \mathrm{mM}$ sodium phosphate buffer containing

$1770.1 \%$ sodium diethyldithiocarbamate and a mixture of ${ }^{13} \mathrm{C}_{6}$ isotopically labelled internal standards (Olchemim, Olomouc, Czech Republic). After centrifugation (20 000 g, $15 \mathrm{~min}, 4^{\circ} \mathrm{C}$ ), the supernatant was transferred into new Eppendorf tubes. The $\mathrm{pH}$ was then adjusted to 2.5 with $1 \mathrm{M} \mathrm{HCl}$ and samples were immediately applied to preconditioned solid-phase extraction columns (Oasis HLB, $30 \mathrm{mg}$ of $1 \mathrm{ml}$; Waters Inc., Milford, MA, USA). After sample application, each column was rinsed with $2 \mathrm{ml} 5 \%$ methanol. Compounds of interest were subsequently eluted with $2 \mathrm{ml}$ of $80 \%$ methanol. UHPLC-MS/MS analysis was performed according to the method described in (Pencik et al., 2018), using an LC-MS/MS system consisting of a 1290 Infinity Binary LC System coupled to a 6490 Triple Quad LC/MS System with Jet Stream and 
quantification was carried out in Agilent MassHunter Workstation Quantitative Analysis software (Agilent Technologies, Santa Clara, CA, USA).

\section{Quantification of JA, JA-lle, SA and ABA}

Samples were extracted, purified and analysed according to a method described in (Simura et al., 2018). Briefly, around $20 \mathrm{mg}$ of frozen material per sample was homogenized and extracted in $1 \mathrm{~mL}$ of ice-cold $50 \%$ aqueous acetonitrile $(\mathrm{v} / \mathrm{v})$ containing a mixture of $\left[{ }^{13} \mathrm{C}\right],\left[{ }^{15} \mathrm{~N}\right]$ or $\left[{ }^{2} \mathrm{H}\right]$ isotopically labelled internal standards using a bead mill $\left(27 \mathrm{~Hz}, 10 \mathrm{~min}, 4^{\circ} \mathrm{C}\right.$; MixerMill, Retsch $\mathrm{GmbH}$, Haan, Germany) and sonicator $\left(3 \mathrm{~min}, 4^{\circ} \mathrm{C}\right.$; Ultrasonic bath P $310 \mathrm{H}$, Elma, Germany). After centrifugation (14000 RPM, $15 \mathrm{~min}, 4^{\circ} \mathrm{C}$ ), the supernatant was purified as follows. A solid-phase extraction column Oasis HLB (30 mg 1 cc, Waters Inc., Milford, MA, USA) was conditioned with $1 \mathrm{ml}$ of $100 \%$ methanol and $1 \mathrm{ml}$ of deionized water (Milli-Q, Merck Millipore, Burlington, MA, USA). After the conditioning steps, each sample was loaded on a SPE column and the flow-through fraction was collected as well as the $1 \mathrm{ml} 30 \%$ aqueous acetonitrile $(\mathrm{v} / \mathrm{v})$ elution fraction. Samples were evaporated to dryness using a SpeedVac SPD111V (Thermo Scientific, Waltham, MA, USA). Prior to LC-MS analysis, samples were dissolved in $40 \mu \mathrm{L}$ of $30 \%$ acetonitrile $(\mathrm{v} / \mathrm{v})$ and transferred to insert-equipped vials. Mass spectrometry analysis of targeted compounds was performed using an UHPLC-ESI-MS/MS system comprising a 1290 Infinity Binary LC system coupled to a 6490 Triple Quad LC/MS system with Jet Stream and Dual Ion Funnel technologies (Agilent Technologies, Santa Clara, CA, USA). The quantification was carried out in Agilent MassHunter Workstation Quantitative Analysis software (Agilent Technologies, Santa Clara, CA, USA).

\section{Phenotypic and statistical analyses}

For root and hypocotyl phenotyping, vertically grown plates were imaged using Epson Perfection V600 photo scanners. For petiole length determination, horizontally grown plates were photographed from above. Lengths were measured from scaled images using FIJI software (Schindelin et al., 2012).

Statistically significant differences between mean values from wild type and mutants were analysed by a two-tailed Student's $t$-test. Multiple comparisons of genotypes, treatments and times were performed by one-way ANOVA followed by Tukey's post hoc test to a 95\% confidence level. Analyses were carried out in GraphPad Prism 6.01.

\section{Accession numbers}

221 ACTIN2 (At3g18780), GH3.1 (At2g14960), GH3.2 (At4g37390), GH3.3 (At2g23170), GH3.4 
(At1g59500), GH3.5 (At4g27260), GH3.6 (At5g54510), GH3.9 (At2g47750), GH3.17

223 (At1g28130).

\section{RESULTS}

Arabidopsis group II GH3s are required for vegetative and reproductive development are known to redundantly modulate IAA levels by catalysing the formation of IAA-amino acid conjugates, which are inactive IAA forms (Staswick et al., 2005; Chen et al., 2010; Porco et al., 2016). Due to functional redundancy, single gh3 mutants often show mild or no phenotypes (Staswick et al., 2005). To bypass such redundancy, we generated a gh3.1,2,3,4,5,6,9,17 octuple mutant (hereafter referred to as $g h 3 o c t$ ) by crossing the individual insertional lines (Fig. S1a, Table S1). To verify that gh3oct was an octuple knock-out, we performed RT-qPCR analysis. We showed that expression of the full-length GH3 transcript was completely abolished by the insertions in the gh3oct mutant, with just marginal expression of GH3.9 in reproductive tissues (Fig. S1b). Because the average number of T-DNAs in insertional lines has been estimated to be 2.1 (Wilson-Sanchez et al., 2014), the gh3oct mutant genome likely harboured several additional insertions that could have jeopardized our interpretations on the GH3-related functions. To rule out this possibility, we sequenced the gh3oct genome and followed a tagged-sequence strategy to map the positions of the insertions. We confirmed the presence of eight insertions within the corresponding $\mathrm{GH} 3$ coding sequences and found two extra insertions at intergenic regions (Fig. S2). Due to their position, the latter were considered unlikely to contribute to the gh3oct phenotypes.

We then explored the developmental consequences of a group-II GH3 knock-out. We found that primary roots from gh3oct mutant seedlings were comparable in length to those of the wild type, whereas lateral root density was notably increased (Fig. 1a,b,j,k). Such a root phenotype was observed along the vegetative phase (Fig. 1j,k). The rosettes from gh3oct seedlings showed classical high-auxin phenotypes, such as epinastic cotyledons (Fig. 1a,b) and elongated hypocotyls (Fig. 1a,b,I) and petioles (Fig. 1c,d,m). The gh3oct mutant also showed photoperiod-independent early flowering (Fig. 1e, S3). Most of the siliques in the gh3oct stems did not elongate properly (Fig. 1i), likely causing a delayed inflorescence arrest (Ware et al., 2020), and thus making gh3oct plants taller (Fig. 1h). We observed the formation of several aberrant flowers that developed into siliques with unfused valves in the gh3oct mutant (Fig. 1f,g), although this phenotype showed incomplete penetrance. Taken together, 
stage and that functional group II GH3s redundantly control the progress and timing of several developmental processes throughout the plant's life cycle.

\section{IAA metabolism in the absence of functional group II GH3s}

Due to the involvement of group II GH3s in IAA metabolism, we explored the concentrations of IAA, the irreversible IAA conjugates IAA-Asp and IAA-Glu, and the IAA inactive forms oxIAA, oxIAA-glc and IAA-glc in shoots and roots from Col-0 and the gh3oct mutant. According to the high-auxin phenotypes displayed by gh3oct plants (Fig. 1), IAA contents were found to be increased in gh3oct seedling shoots (Fig. 2). However, total IAA in root tissues was found at wild type levels (Fig. 2). Despite these modest differences in IAA contents, the roots of gh3oct seedlings were hypersensitive to exogenous IAA (Fig. S4). As expected for a total knock-out of the IAA-amido synthetase functions, no detectable IAA-Asp was found in gh3oct plants (Fig. 2). Surprisingly, decreased yet still detectable amounts of IAA-Glu were found in shoots and unchanged IAA-Glu levels were detected in roots from gh3oct seedlings (Fig. 2). The contents of the oxidative catabolites oxIAA and oxIAA-glc were diminished in gh3oct shoots and roots (Fig. 2), whereas no differences in the content of IAA-glc were found (Fig. 2). Overall, our data suggested that gh3oct plants have a reduced ability to inactivate IAA and that IAA inactivation, by means of conjugation to Glu, could still occur in the absence of functional group-II GH3 members. Our data additionally indicated a connection between the production of IAA-aa conjugates and IAA oxidative catabolites.

\section{gh3oct mutant tolerance to salinity and osmotic stress}

GH3-mediated IAA metabolism has previously been suggested to modulate abiotic stress responses in plants (Park et al., 2007; Du et al., 2012; Kirungu et al., 2019). Therefore, we focused on the involvement of group II GH3s in the response to salinity. When examining transcriptomic datasets from Arabidopsis plants exposed to $\mathrm{NaCl}$, we found that Arabidopsis group II GH3s are responsive to salt treatments (Fig. S5). To corroborate this response under our experimental conditions, we used RT-qPCR to determine the relative expression of each $\mathrm{GH} 3$ gene to $\mathrm{NaCl}$ in seedling roots. We found all root-expressed group II GH3s to be upregulated upon $\mathrm{NaCl}$ application (Fig. 3a), suggesting that these enzymes might play a role in the response to salinity.

Salinity is well-known to cause general arrest in plant growth (Zhao et al., 2020), and a decreased length of the primary root in salt-stressed Arabidopsis plants is documented (Smolko et al., 2021). Thus, we used the growth of the primary root as an indicator of the 
290 tolerance to salinity stress. Compared to Col-0 wild type, gh3oct plants were more tolerant to

$29150 \mathrm{mM} \mathrm{NaCl}$ (Fig. 3b,c). The gh3oct mutant also grew better than Col-0 at higher $\mathrm{NaCl}$

292 concentrations (Fig. 3c). Besides $\mathrm{NaCl}$, we found that the gh3oct mutant exhibited higher

293 tolerance to other osmotic stressors, such as sorbitol and mannitol, over the vegetative phase

294 (Fig. S6). Thus, the data suggested that genetic disruption of the group II GH3s confers salinity

295 tolerance, likely as part of a general osmotolerant mechanism.

296

297

\section{Tolerance to salinity in the gh3oct mutant is related to endogenous IAA content}

To investigate the relevance of the elevated endogenous IAA content in gh3oct plants for the tolerance to salinity, we co-treated gh3oct seedlings with both $\mathrm{NaCl}$ and IAA synthesis inhibitors. As shown in Fig. 3d, the higher tolerance to $\mathrm{NaCl}$ of the gh3oct mutant, in terms of a higher root growth ratio, was abolished by addition of either yucasin or PPBo, which are two inhibitors of YUCCA enzymes that cause a decrease in plant IAA levels by competing with the IAA precursor IPyA (Nishimura et al., 2014; Kakei et al., 2015). To rule out the possibility that the decreased root growth ratio was caused by the effect of the IAA synthesis inhibitors on root growth rather than the tolerance to salinity, we inspected the effects of yucasin and PPBo on the root growth of gh3oct seedlings in the absence of $\mathrm{NaCl}$. We found that, at the employed concentrations, none of the inhibitors caused a decrease in the length of the root, whereas both decreased the lateral root density of the gh3oct mutant (Fig. 3e,f).

We next explored the contents of IAA and IAA metabolites and the tolerance to salinity of the gh3 single mutants. IAA levels were found to be comparable to the wild type in all gh3 mutant shoots and roots, except for gh3.1, which showed decreased IAA levels in roots, and gh3.2, which showed increased IAA levels in shoots but decreased levels in roots (Fig. S7). The IAA-Asp concentration was slightly decreased in gh3.3 shoots and gh3.6 roots, while a reduced concentration of IAA-Glu was detected in gh3.1 and gh3.2 roots (Fig. S7). Levels of oxIAA were decreased in gh3.2 shoots but were comparable to the wild type in the other gh3 mutant shoots and roots (Fig. S7). Under our experimental conditions, only gh3.5 plants showed salt-tolerant root growth (Fig. S8a,b). However, different from the gh3oct mutant, the tolerance to salinity of $g h 3.5$ plants was not suppressed by decreasing endogenous IAA levels (Fig. S8c). Taken together, the results indicated that the tolerance to salinity stress of the gh3oct mutant is related to their higher endogenous IAA contents. 
323 Salinity and drought are initially perceived by the plant roots as an osmotic stress. Therefore,

324 it is not uncommon for salt-tolerant genotypes to also exhibit a degree of tolerance to drought

325 stress and vice versa (Uddin et al., 2016; Lamers et al., 2020). To test whether gh3oct plants

326 better tolerated drought, we first grew Col-0 and gh3oct seedlings on media plates with a

327 reduced water potential by using polyethylene glycol (PEG) perfusion (Verslues et al., 2006).

328 The root growth ratio of gh3oct plants grown on PEG-perfused plates was higher than that of 329 the wild type (Fig. 4a), suggesting that the gh3oct mutant was more tolerant to drought stress 330 when grown in vitro. Because gh3.5 plants also showed more tolerant growth under salinity, we investigated the growth ratio of gh3.5 roots in PEG-perfused media. In contrast to the gh3oct mutant, gh3.5 plants were as sensitive as the wild type to PEG-mediated reduction of the water potential (Fig. S8d).

Finally, we explored whether the drought tolerance of the gh3oct mutant could be reproduced when grown on soil. We planted stratified Col-0 and gh3oct seeds in well-watered soil pots and then withheld irrigation until the plants manifested drought symptoms. As shown in Fig. 4b, gh3oct mutant plants showed milder drought symptoms and a higher survival rate after re-watering ( $80 \%$ in gh3oct vs. $53 \%$ in Col-0). To rule out that pots containing gh $30 c t$ plants could retain more water, we compared the weight of well-watered to that of dry pots (before drought symptoms appeared) for both genotypes. As shown in Fig. 4c, we found no differences between Col- 0 and gh3octpots. Thus, our results further supported that the gh3oct mutant was more tolerant to soil drought.

\section{Hormonal landscape in gh3oct plants under salinity}

In addition to IAA, group II GH3 members have been shown to conjugate amino acids to other phytohormones, such as jasmonic acid (JA) and salicylic acid (SA) (Gutierrez et al., 2012; Westfall et al., 2016). Given the involvement of GH3s in the response to salinity, we quantified the endogenous contents of these phytohormones in plants grown in control and $\mathrm{NaCl}$-supplemented media. We found higher IAA levels in gh3oct mutant seedlings grown at

3500 and $75 \mathrm{mM} \mathrm{NaCl}$, whereas the IAA concentration was comparable to Col-0 at $150 \mathrm{mM} \mathrm{NaCl}$ 351 (Fig. 5). Levels of JA, the JA bioactive form JA-lle and SA were similar in Col-0 and gh3oct seedlings under both control and salinity conditions (Fig. 5). JA and JA-lle levels increased in both Col-0 and gh3oct plants grown on $\mathrm{NaCl}$-supplemented media (Fig. 5). Because $\mathrm{NaCl}$ stress is known to trigger an increase in levels of abscisic acid (ABA) (Simura et al., 2018), we also quantified levels of this phytohormone. ABA content was higher in gh3oct seedlings 
grown in the control medium, but this difference disappeared in seedlings that germinated under salinity (Fig. 5).

Next, we explored changes in endogenous levels of IAA, JA, JA-Ile, SA and ABA in response to salinity. For that, we transferred Col-0 and gh3oct seedlings to media plates supplemented with $150 \mathrm{mM} \mathrm{NaCl}$ and quantified the phytohormone concentrations at 6 and $24 \mathrm{~h}$ upon exposure to $\mathrm{NaCl}$. IAA levels were higher in gh3oct seedlings at all inspected times under control conditions, but they became comparable to those of Col-0 in response to 150 $\mathrm{mM} \mathrm{NaCl}$ (Fig. S9). This result is in accordance with the similar IAA contents in Col-0 and gh3oct seedlings grown at $150 \mathrm{mM} \mathrm{NaCl}$ concentration (Fig. 5). Levels of JA and JA-Ile were neither affected by genotype nor by salinity (Fig. S9). Levels of SA were significantly lower in gh3oct seedlings after $6 \mathrm{~h}$ of exposure to salinity, although this difference vanished after $24 \mathrm{~h}$ (Fig. S9). Endogenous levels of ABA prominently increased in both genotypes in response to salinity. However, the ABA content was higher in the gh3oct mutant after $24 \mathrm{~h}$ of exposure to $\mathrm{NaCl}$ (Fig. S9). Because higher ABA levels were also found in gh3oct plants grown under control conditions (Fig. 5) and ABA is known to modulate the response to osmotic stresses, e.g. salinity, by mediating stomatal closure (Hedrich \& Shabala, 2018), we hypothesized a higher ability of the gh3oct mutant to deal with water loss. However, our observations indicated that the water loss rate in gh3oct detached rosettes was indistinguishable from that of the wild type (Fig. S10).

In summary, we found altered levels of IAA and $A B A$ under control conditions and of $S A$ and $A B A$ in response to salinity in gh3oct mutant plants.

\section{DISCUSSION}

\section{IAA metabolism and plant development without functional group II GH3s}

Conjugation of IAA to amino acids is an important metabolic pathway regulating levels of this bioactive auxin and is performed by group II GH3 proteins (Staswick et al., 2005). Studies on single gh3 mutants have demonstrated the relevance of IAA conjugation for plant developmental processes, such as root meristem size control (Di Mambro et al., 2017) and hypocotyl elongation (Zheng et al., 2016). To identify additional roles of the group II GH3s in auxin metabolism and plant development, we generated an octuple group II GH3 mutant. Our phenotypical analyses of gh3oct mutant plants supported redundant involvement of group II GH3s in lateral root, shoot and fruit development, as well as in vegetative-to-reproductive transition. While our data were not sufficient to support a direct causality between IAA metabolism and early flowering or aberrant fruit development in gh3oct plants, the observed 
increased lateral root density and longer hypocotyls and petioles are well-known high-auxin phenotypes (Sugawara et al., 2009; Gao et al., 2020). Our analyses found that the IAA content was only slightly increased in gh3oct shoots but at wild-type levels in roots. This suggests that the high-auxin phenotypes displayed by gh3octplants might arise from highly spatiotemporally localized IAA increases, which do not remarkably alter the total IAA content in shoot and root tissues where other mechanisms of IAA control may be more important.

We previously reported a sextuple GH3 knockout mutant in which 6 group II GH3s (GH3.16) were disrupted (Porco et al., 2016). No detectable levels of IAA-Asp were found in the gh3 sextuple mutant, whereas conjugation of IAA to Glu was upregulated. This was likely attributable to the activity of GH3.17, which is highly specific for the formation of IAA-Glu (Staswick et al., 2005; Brunoni et al., 2019). Therefore, one might expect that formation of both IAA-Asp and IAA-Glu would be abolished after knocking out all group II GH3s. However, the presence of detectable IAA-Glu content in gh3oct seedlings, which displayed null expression of group-II GH3 genes, strongly suggests that additional GH3 or GH3-like enzymes work in IAA inactivation by means of conjugation to glutamate in Arabidopsis.

DAO proteins have been shown to regulate IAA levels by mediating its metabolic inactivation through oxidation (Zhao et al., 2013; Porco et al., 2016; Zhang et al., 2016). Levels of the oxidative IAA catabolite oxIAA are known to rapidly increase in response to endogenous or exogenous IAA increases (Kubes et al., 2012; Pencik et al., 2013). However, despite showing locally increased IAA levels, as inferred from their multiple high-auxin phenotypes and hypersensitivity to IAA, reduced levels of oxIAA and oxIAA-glc were observed in gh3oct plants. DAOs and GH3s have been suggested to establish parallel and redundant pathways for IAA inactivation, with the GH3 pathway playing a compensatory function, as supported by increased and decreased conjugate levels in the DAO1 mutant and overexpressing plants, respectively (Porco et al., 2016; Zhang et al., 2016). This model has recently been challenged by the finding that IAA-Asp is an in vivo substrate for DAO1 oxidase, which additionally accounts for the formation of oxIAA-Asp conjugates (Müller et al., 2021). In a context where DAOs function downstream of group II GH3s, the reduced levels of oxIAA and oxIAA-glc in gh3oct plants found in the present work suggest that a significant fraction of the plant oxIAA pool derives from oxIAA-aa conjugates.

\section{Cooperative involvement of group II GH3s in responses to salinity and drought}

Salinity and drought are major constraints on plant growth, and therefore represent serious threats to agriculture and forestry. Data from several studies over the past decade support a 
424 link between auxin and plant responses to salinity (Wang et al., 2009; Liu et al., 2015; Korver 425 et al., 2018; Fu et al., 2019) and drought stress (Zhang et al., 2009; Zhang et al., 2012; Shi et 426 al., 2014; Jung et al., 2015; Zhang et al., 2020). Spatiotemporal auxin distribution and auxin 427 sensing modulate adaptive growth responses to $\mathrm{NaCl}$ stress (Wang et al., 2009; Liu et al., 428 2015). Recently, Aux/IAA auxin co-receptors were shown to be required for plant tolerance to 429 water deficit, thus suggesting a central role for auxin in integrating drought signals to elaborate 430 genetic and physiological responses (Shani et al., 2017). Although the pathways that mediate auxin-mediated responses to salinity and drought are only just being elucidated, it appears that auxin may mediate tolerance to water deprivation by regulating several adaptive responses leading to stress avoidance. These include (i) adaptive root growth (Leftley et al., 2021), (ii) ROS scavenging (Fu et al., 2019), (iii) regulation of stomatal apertures (Salehin et al., 2019), and (iv) crosstalk with ABA and other hormones involved in the responses to salinity and drought (Sun \& Li, 2014; Yu et al., 2020; Salvi et al., 2021).

437 Our work revealed that gh3oct mutant plants showed more tolerant growth under salinity, and that this tolerance was related to endogenous IAA levels. Tolerance to salinity was also higher in gh3.5 mutant plants, although this effect appeared to be unaffected by endogenous IAA levels. GH3.5 is well-known to mediate the conjugation of other plant hormones, including SA and JA (Gutierrez et al., 2012; Westfall et al., 2016), suggesting that the salt-tolerant growth of gh3.5 roots may rather be related to the SA or JA pathways. The increased root branching of gh3oct plants without penalty for primary root growth, produced a more robust root system, which might contribute to the better performance of gh3oct plants under water stress conditions.

446 In addition to root system architecture, the increased tolerance of gh3oct plants to salinity and 447 drought might be driven by increased $A B A$ content. $A B A$ is a stress-related hormone whose 448 levels increase very rapidly upon salt and drought stress sensing and is well-known for its 449 direct involvement in the response to osmotic stresses, primarily by mediating stomatal 450 closure (Hedrich \& Shabala, 2018), although other stomata-independent mechanisms have 451 been reported (Thalmann et al., 2016). The increased ABA content but wild-type water loss 452 rate in gh3oct plants grown in control conditions indeed rule out a predisposition for gh3oct 453 plants to tolerate water deficit based on lower transpiration, but suggests that other ABAmediated mechanisms might assist gh3oct plants to cope with this stress. 
457 In this work, we present a group-II GH3 knock-out as a useful genetic tool to research 458 metabolic pathways regulating levels of the main auxin IAA. It has long been accepted that 459 group II GH3 members carry out conjugation of IAA to amino acids. Our metabolic analyses 460 in gh3oct plants indicated that additional GH3s or GH3-like proteins might modulate IAA levels 461 by means of conjugation to glutamate. Additionally, our data suggested that the major IAA 462 catabolite oxIAA is, at least in part, produced from the GH3 pathway.

463 Examination of mutant gh3oct plants also revealed that plant tolerance to water deprivation 464 stresses, such as salinity and drought, is modulated by redundant auxin conjugation. 465 Identifying as many components as possible of a plant's responses to salinity and drought is 466 important for suggesting targets to guide the design of chemical and/or gene-editing 467 approaches directed to enhance stress tolerance in plants. While the mechanisms behind the 468 auxin-related tolerance to salinity and drought remain to be elucidated, the present work 469 supports strategies based on salinity- and drought-inducible disruption of group II GH3s as a 470 profitable tool to engineer plants that better tolerate water stress.

471

472 


\section{FIGURE LEGENDS}

Figure 1. Phenotypes of the gh3oct mutant. (a-e) Col-0 and gh3oct plants at (a,b) 7, (c,d) 20 and (d) 23 days after stratification (das). ( $f, g)$ Aberrant reproductive structures occasionally formed by gh3oct mutant from latest inflorescences. (h) Plant height of Col-0 and gh3oct flowering plants. (i) Stems from Col-0 and the gh3oct mutant. White arrowheads indicate siliques that did not elongate further in the gh3oct mutant. (j,k) Primary root length (j) and lateral root density (k) determined at 5, 7, 10 and 12 das. (I) Hypocotyl length determined at 10 das. $(\mathrm{m})$ Petiole length determined at 15 das. Scale bars indicate $(a, b) 2 \mathrm{~mm},(\mathrm{c}, \mathrm{d}) 1 \mathrm{~cm}$, $(\mathrm{f}, \mathrm{g}) 2 \mathrm{~mm}$, and (i) $2 \mathrm{~cm}$. Asterisks indicate values statistically different from Col-0 in a twotailed Student's $t$-test $\left[{ }^{*} p<0.05,{ }^{* * * *} p<0.0001 ;\right.$ (j,k) $n \geq 17$, (I) $n \geq 83$, and (m) $\left.n=20\right]$.

Figure 2. Levels of IAA and IAA metabolites in shoots and roots of the gh3oct mutant. Concentration (expressed in picomoles per gram of fresh weight) of indole-3-acetic acid (IAA), indole-3-acetyl-aspartate (IAA-Asp), indole-3-acetyl-glutamate (IAA-Glu), 2-oxindole-3-acetic acid (oxIAA), 1-O-(2-oxindole-3-ylacetyl)- $\beta$-d-glucose (oxIAA-glc) and 1-O-indole-3-ylacetyl$\beta$-d-glucose (IAA-glc) in shoots and roots from 7-day-old Col-0 (black bars) and gh3oct (red bars) seedlings. Error bars indicate standard deviation. Asterisks indicate values significantly different from Col-0 in a two-tailed Student's $t$-test $\left({ }^{*} p<0.05,{ }^{* * *} p<0.001 ; n=5\right)$.

Figure 3. Group II GH3s are upregulated upon salt treatment and the gh3oct mutant showed IAA-dependent tolerance to salinity. (a) Transcriptional response of group II GH3 genes from 10-day-old Col-0 roots determined by qRT-PCR 6 hours after transferring to MS media supplemented with 0,75 or $150 \mathrm{mM} \mathrm{NaCl}$. No expression of GH3.4 and GH3.9 was detected in any sample. Error bars indicate standard deviation. (b) Seedlings from 7-day-old Col-0 and gh3oct transferred at day 5 to control MS plates or MS plates supplemented with $50 \mathrm{mM} \mathrm{NaCl}$. (c) Primary root length of Col-0 and gh3oct seedlings grown for 7 days at different concentrations of $\mathrm{NaCl}$. (d) Root growth ratio of 4-day-old seedlings transferred for another 3 days to media plates containing $50 \mathrm{mM} \mathrm{NaCl}, 50 \mathrm{mM} \mathrm{NaCl}+20 \mu \mathrm{M}$ of the YUCCA inhibitor yucasin or $50 \mathrm{mM} \mathrm{NaCl}+1 \mu \mathrm{M}$ of the YUCCA inhibitor PPBo (4phenoxyphenylboronic acid). To calculate the ratio, the length of the root from seedlings grown on $\mathrm{NaCl}, \mathrm{NaCl}+$ yucasin and $\mathrm{NaCl}+$ PPBo plates was divided by the mean root length of seedlings grown on control MS, MS + yucasin and MS + PPBo plates, respectively. (e, f) Root length (e) and lateral root density (f) in 7-day-old gh3oct mutant seedlings grown on control MS plates, and on plates supplemented with $20 \mu \mathrm{M}$ of yucasin or $1 \mu \mathrm{M}$ of PPBo. Asterisks indicate (a) $\triangle$ CT values significantly different from MS $(n=4),(c, d)$ values significantly different from Col-0 $(n \geq 11)$, (e,f) values significantly different from MS $(n \geq 17)$ in a two-tailed Student's $t$-test $\left({ }^{*} p<0.05 ;{ }^{* *} p<0.01,{ }^{* * *} p<0.001,{ }^{* * *} p<0.0001\right)$.

Figure 4. gh3oct plants are tolerant to drought. (a) Root growth ratio of Col-0 and gh3oct seedlings in media with lowered water potential. 4-day-old seedlings were transferred to plates perfused with a solution of PEG8000 at the indicated concentration and grown for another 6 days. To calculate the ratio, the root length from seedlings in PEG-containing plates was divided by the mean root length of seedlings grown on control plates (perfused with liquid MS). Asterisks indicate statistical significantly different growth ratios from Col-0 in a two-tailed Student's $t$-test ${ }^{* * *} p<0.001 ; n \geq 25$ ). (b) Tolerance of Col-0 and gh3oct plants to drought stress on soil. The image corresponds to 3 -week-old plants on dry soil. Survival rate after rewatering is indicated at the bottom and represents the mean percentage from 3 independent experiments with 27, 36 and 23 plants per genotype. (c) Soil pot weight in the drought experiment. Pots were prepared with $40 \mathrm{~g}$ of soil mixture, watered and weighed at the beginning of the experiment (initial). Pots were re-weighed when the soil was noticeably dry and before plants started showing drought symptoms (before drought). No significant differences were found between Col-0 and gh3oct pots in a two-tailed Student's $t$-test. 
527 Figure 5. Phytohormone levels from Col-0 and the gh3oct mutant grown under different

$528 \mathrm{NaCl}$ concentrations. Concentrations, expressed in picomoles per gram of fresh weight, of 529 indole-3-acetic acid (IAA), jasmonic acid (JA), jasmonic acid-isoleucine (JA-lle), salicylic acid 530 (SA) and abscisic acid (ABA) in Col-0 and gh3oct seedlings were determined 7 days after germination on plates containing 0,75 or $150 \mathrm{mM} \mathrm{NaCl}$. Error bars indicate standard deviation. Asterisks indicate values significantly different from the corresponding values of Col-0 in a two-tailed Student's $t$-test $\left({ }^{*} p<0.05,{ }^{* *} p<0.01,{ }^{* *} p<0.001 ; n=4\right)$. <LOD: below the limit of detection.

\section{SUPPORTING INFORMATION}

Figure S1. The gh3oct mutant is an octuple knock-out.

Figure S2. The gh3oct mutant harbours two extra T-DNA insertions at intergenic regions.

Figure S3. The gh3oct is a photoperiod-independent early flowering mutant.

Figure S4. Root growth in the gh3oct mutant is hypersensitive to IAA.

Figure S5. Transcriptional response of group-II GH3 genes to salinity according to published datasets.

Figure S6. Root growth in the gh3oct mutant is tolerant to different osmolytes.

Figure S7. Levels of IAA and IAA metabolites in shoots and roots from group-II gh3 single mutants.

Figure S8. Salinity and drought tolerance of $g h 3$ single mutants.

Figure S9. Levels of IAA and stress-related phytohormones in response to salinity in the gh3oct mutant.

552 Table S1. Insertion lines combined to obtain the gh3oct mutant.

553 Table S2. Primer sets used in this work. 


\section{ACKNOWLEDGMENTS}

Research in the laboratory of Karin Ljung is supported by grants from the Swedish Foundation for Strategic Research (Vinnova), the Knut and Alice Wallenberg Foundation (KAW), the Swedish research councils VR and Formas. E.M.-B. (JCK-1811) held postdoctoral fellowship from Kempestiftelserna. A.P. and O.N. were financially supported by the Ministry of Education, Youth and Sports of the Czech Republic (European Regional Development FundProject "Plants as a tool for sustainable global development" No. CZ.02.1.01/0.0/0.0/16_019/0000827). We also acknowledge the Swedish Metabolomics Centre (http://www.swedishmetabolomicscentre.se/) for access to instrumentation.

\section{AUTHOR CONTRIBUTIONS}

E.M.-B., R.C.-S. and K.L. conceived and designed the research; E.M.-B. and R.C.-S. performed most of the experiments; J.Š. and A.P. performed hormone analyses; P.S. made the gh3oct mutant; R.C.-S. prepared the manuscript draft; E.M.-B. and R.C.-S. wrote the manuscript with input from all authors. This research was supported by funds to K.L. 


\section{REFERENCES}

Band LR. 2021. Auxin fluxes through plasmodesmata. New Phytol 231(5): 1686-1692.

Bottcher C, Boss PK, Davies C. 2011. Acyl substrate preferences of an IAA-amido synthetase account for variations in grape (Vitis vinifera L.) berry ripening caused by different auxinic compounds indicating the importance of auxin conjugation in plant development. J Exp Bot 62(12): 4267-4280.

Brunoni F, Collani S, Casanova-Saez R, Simura J, Karady M, Schmid M, Ljung K, Bellini C. 2020. Conifers exhibit a characteristic inactivation of auxin to maintain tissue homeostasis. New Phytol 226(6): 1753-1765.

Brunoni F, Collani S, Simura J, Schmid M, Bellini C, Ljung K. 2019. A bacterial assay for rapid screening of IAA catabolic enzymes. Plant Methods 15: 126.

Casanova-Saez R, Mateo-Bonmati E, Ljung K. 2021. Auxin Metabolism in Plants. Cold Spring Harb Perspect Biol 13(3).

Casanova-Saez R, Voss U. 2019. Auxin Metabolism Controls Developmental Decisions in Land Plants. Trends Plant Sci 24(8): 741-754.

Chen Q, Westfall CS, Hicks LM, Wang S, Jez JM. 2010. Kinetic basis for the conjugation of auxin by a GH3 family indole-acetic acid-amido synthetase. J Biol Chem 285(39): 29780-29786.

Di Mambro R, De Ruvo M, Pacifici E, Salvi E, Sozzani R, Benfey PN, Busch W, Novak O, Ljung K, Di Paola L, et al. 2017. Auxin minimum triggers the developmental switch from cell division to cell differentiation in the Arabidopsis root. Proc Natl Acad Sci U S A 114(36): E7641-E7649.

Du H, Wu N, Fu J, Wang S, Li X, Xiao J, Xiong L. 2012. A GH3 family member, OsGH3-2, modulates auxin and abscisic acid levels and differentially affects drought and cold tolerance in rice. J Exp Bot 63(18): 6467-6480.

Fu Y, Yang Y, Chen S, Ning N, Hu H. 2019. Arabidopsis IAR4 Modulates Primary Root Growth Under Salt Stress Through ROS-Mediated Modulation of Auxin Distribution. Front Plant Sci 10: 522.

Gallei M, Luschnig C, Friml J. 2020. Auxin signalling in growth: Schrodinger's cat out of the bag. Curr Opin Plant Biol 53: 43-49.

Gao Y, Dai X, Aoi Y, Takebayashi Y, Yang L, Guo X, Zeng Q, Yu H, Kasahara H, Zhao Y. 2020. Two homologous INDOLE-3-ACETAMIDE (IAM) HYDROLASE genes are required for the auxin effects of IAM in Arabidopsis. J Genet Genomics 47(3): 157-165.

Gutierrez L, Mongelard G, Flokova K, Pacurar DI, Novak O, Staswick P, Kowalczyk M, Pacurar M, Demailly H, Geiss G, et al. 2012. Auxin controls Arabidopsis adventitious root initiation by regulating jasmonic acid homeostasis. Plant Cell 24(6): 2515-2527.

Hammes UZ, Murphy AS, Schwechheimer C. 2021. Auxin Transporters-A Biochemical View. Cold Spring Harb Perspect Biol.

Hanania U, Velcheva M, Sahar N, Perl A. 2004. An improved method for isolating high-quality DNA fromVitis vinifera nuclei. Plant Molecular Biology Reporter 22(2): 173-177.

Hedrich R, Shabala S. 2018. Stomata in a saline world. Curr Opin Plant Biol 46: 87-95.

Jung H, Lee DK, Choi YD, Kim JK. 2015. OsIAA6, a member of the rice Aux/IAA gene family, is involved in drought tolerance and tiller outgrowth. Plant Sci 236: 304-312.

Kai K, Horita J, Wakasa K, Miyagawa H. 2007. Three oxidative metabolites of indole-3-acetic acid from Arabidopsis thaliana. Phytochemistry 68(12): 1651-1663. 
Kakei Y, Yamazaki C, Suzuki M, Nakamura A, Sato A, Ishida Y, Kikuchi R, Higashi S, Kokudo Y, Ishii T, et al. 2015. Small-molecule auxin inhibitors that target YUCCA are powerful tools for studying auxin function. Plant J 84(4): 827-837.

Khan S, Stone JM. 2007. Arabidopsis thaliana GH3.9 influences primary root growth. Planta 226(1): 21-34.

Kirungu JN, Magwanga RO, Lu P, Cai X, Zhou Z, Wang X, Peng R, Wang K, Liu F. 2019. Functional characterization of Gh_A08G1120 (GH3.5) gene reveal their significant role in enhancing drought and salt stress tolerance in cotton. BMC Genet 20(1): 62.

Kojima M, Kamada-Nobusada T, Komatsu H, Takei K, Kuroha T, Mizutani M, Ashikari M, Ueguchi-Tanaka M, Matsuoka M, Suzuki K, et al. 2009. Highly sensitive and highthroughput analysis of plant hormones using MS-probe modification and liquid chromatography-tandem mass spectrometry: an application for hormone profiling in Oryza sativa. Plant Cell Physiol 50(7): 1201-1214.

Korver RA, Koevoets IT, Testerink C. 2018. Out of Shape During Stress: A Key Role for Auxin. Trends Plant Sci 23(9): 783-793.

Kowalczyk M, Sandberg G. 2001. Quantitative analysis of indole-3-acetic acid metabolites in Arabidopsis. Plant Physiol 127(4): 1845-1853.

Kubes M, Yang H, Richter GL, Cheng Y, Mlodzinska E, Wang X, Blakeslee JJ, Carraro N, Petrasek J, Zazimalova E, et al. 2012. The Arabidopsis concentration-dependent influx/efflux transporter $A B C B 4$ regulates cellular auxin levels in the root epidermis. Plant J 69(4): 640-654.

Lamers J, van der Meer T, Testerink C. 2020. How Plants Sense and Respond to Stressful Environments. Plant Physiol 182(4): 1624-1635.

LeClere S, Tellez R, Rampey RA, Matsuda SP, Bartel B. 2002. Characterization of a family of IAA-amino acid conjugate hydrolases from Arabidopsis. J Biol Chem 277(23): 2044620452.

Leftley N, Banda J, Pandey B, Bennett M, Voss U. 2021. Uncovering How Auxin Optimizes Root Systems Architecture in Response to Environmental Stresses. Cold Spring Harb Perspect Biol.

Liu W, Li RJ, Han TT, Cai W, Fu ZW, Lu YT. 2015. Salt stress reduces root meristem size by nitric oxide-mediated modulation of auxin accumulation and signaling in Arabidopsis. Plant Physiol 168(1): 343-356.

Ludwig-Muller J, Julke S, Bierfreund NM, Decker EL, Reski R. 2009. Moss (Physcomitrella patens) GH3 proteins act in auxin homeostasis. New Phytol 181(2): 323-338.

Lup SD, Wilson-Sanchez D, Andreu-Sanchez S, Micol JL. 2021. Easymap: A User-Friendly Software Package for Rapid Mapping-by-Sequencing of Point Mutations and Large Insertions. Front Plant Sci 12: 655286.

Mateo-Bonmati E, Casanova-Saez R, Simura J, Ljung K. 2021. Broadening the roles of UDPglycosyltransferases in auxin homeostasis and plant development. New Phytol.

Müller K, Dobrev PI, Pěnčík A, Hošek P, Vondráková Z, Filepová R, Malínská K, Brunoni F, Helusová L, Moravec T, et al. 2021. DIOXYGENASE FOR AUXIN OXIDATION 1 catalyzes the oxidation of IAA amino acid conjugates. Plant Physiology.

Nishimura T, Hayashi K, Suzuki H, Gyohda A, Takaoka C, Sakaguchi Y, Matsumoto S, Kasahara H, Sakai T, Kato J, et al. 2014. Yucasin is a potent inhibitor of YUCCA, a key enzyme in auxin biosynthesis. Plant $J$ 77(3): 352-366.

Novak O, Henykova E, Sairanen I, Kowalczyk M, Pospisil T, Ljung K. 2012. Tissue-specific profiling of the Arabidopsis thaliana auxin metabolome. Plant J 72(3): 523-536. 
Ostin A, Kowalyczk M, Bhalerao RP, Sandberg G. 1998. Metabolism of indole-3-acetic acid in Arabidopsis. Plant Physiol 118(1): 285-296.

Park JE, Park JY, Kim YS, Staswick PE, Jeon J, Yun J, Kim SY, Kim J, Lee YH, Park CM. 2007. GH3-mediated auxin homeostasis links growth regulation with stress adaptation response in Arabidopsis. J Biol Chem 282(13): 10036-10046.

Pencik A, Casanova-Saez R, Pilarova V, Zukauskaite A, Pinto R, Micol JL, Ljung K, Novak O. 2018. Ultra-rapid auxin metabolite profiling for high-throughput mutant screening in Arabidopsis. J Exp Bot 69(10): 2569-2579.

Pencik A, Rolcik J, Novak O, Magnus V, Bartak P, Buchtik R, Salopek-Sondi B, Strnad M. 2009. Isolation of novel indole-3-acetic acid conjugates by immunoaffinity extraction. Talanta 80(2): 651-655.

Pencik A, Simonovik B, Petersson SV, Henykova E, Simon S, Greenham K, Zhang Y, Kowalczyk M, Estelle M, Zazimalova E, et al. 2013. Regulation of auxin homeostasis and gradients in Arabidopsis roots through the formation of the indole-3-acetic acid catabolite 2oxindole-3-acetic acid. Plant Cell 25(10): 3858-3870.

Porco S, Pencik A, Rashed A, Voss U, Casanova-Saez R, Bishopp A, Golebiowska A, Bhosale R, Swarup R, Swarup K, et al. 2016. Dioxygenase-encoding AtDAO1 gene controls IAA oxidation and homeostasis in Arabidopsis. Proc Natl Acad Sci U S A 113(39): 1101611021.

Rampey RA, LeClere S, Kowalczyk M, Ljung K, Sandberg G, Bartel B. 2004. A family of auxinconjugate hydrolases that contributes to free indole-3-acetic acid levels during Arabidopsis germination. Plant Physiol 135(2): 978-988.

Salehin M, Li B, Tang M, Katz E, Song L, Ecker JR, Kliebenstein DJ, Estelle M. 2019. Auxinsensitive Aux/IAA proteins mediate drought tolerance in Arabidopsis by regulating glucosinolate levels. Nat Commun 10(1): 4021.

Salvi P, Manna M, Kaur H, Thakur T, Gandass N, Bhatt D, Muthamilarasan M. 2021. Phytohormone signaling and crosstalk in regulating drought stress response in plants. Plant Cell Rep.

Schindelin J, Arganda-Carreras I, Frise E, Kaynig V, Longair M, Pietzsch T, Preibisch S, Rueden C, Saalfeld S, Schmid B, et al. 2012. Fiji: an open-source platform for biological-image analysis. Nat Methods 9(7): 676-682.

Schmittgen TD, Livak KJ. 2008. Analyzing real-time PCR data by the comparative $C(T)$ method. Nat Protoc 3(6): 1101-1108.

Shani E, Salehin M, Zhang Y, Sanchez SE, Doherty C, Wang R, Mangado CC, Song L, Tal I, Pisanty $O$, et al. 2017. Plant Stress Tolerance Requires Auxin-Sensitive Aux/IAA Transcriptional Repressors. Curr Biol 27(3): 437-444.

Shi H, Chen L, Ye T, Liu X, Ding K, Chan Z. 2014. Modulation of auxin content in Arabidopsis confers improved drought stress resistance. Plant Physiol Biochem 82: 209-217.

Simura J, Antoniadi I, Siroka J, Tarkowska D, Strnad M, Ljung K, Novak O. 2018. Plant Hormonomics: Multiple Phytohormone Profiling by Targeted Metabolomics. Plant Physiol 177(2): 476-489.

Smolko A, Bauer N, Pavlovic I, Pencik A, Novak O, Salopek-Sondi B. 2021. Altered Root Growth, Auxin Metabolism and Distribution in Arabidopsis thaliana Exposed to Salt and Osmotic Stress. Int J Mol Sci 22(15).

Staswick PE, Serban B, Rowe M, Tiryaki I, Maldonado MT, Maldonado MC, Suza W. 2005. Characterization of an Arabidopsis enzyme family that conjugates amino acids to indole-3-acetic acid. Plant Cell 17(2): 616-627. 
Staswick PE, Tiryaki I, Rowe ML. 2002. Jasmonate response locus JAR1 and several related Arabidopsis genes encode enzymes of the firefly luciferase superfamily that show activity on jasmonic, salicylic, and indole-3-acetic acids in an assay for adenylation. Plant Cell 14(6): 1405-1415.

Sugawara S, Hishiyama S, Jikumaru Y, Hanada A, Nishimura T, Koshiba T, Zhao Y, Kamiya Y, Kasahara H. 2009. Biochemical analyses of indole-3-acetaldoxime-dependent auxin biosynthesis in Arabidopsis. Proc Natl Acad Sci U S A 106(13): 5430-5435.

Sun J, Li C 2014. Cross Talk of Signaling Pathways Between ABA and Other Phytohormones. In: Zhang D-P ed. Abscisic Acid: Metabolism, Transport and Signaling. Dordrecht: Springer Netherlands, 243-253.

Takehara S, Sakuraba S, Mikami B, Yoshida H, Yoshimura H, Itoh A, Endo M, Watanabe N, Nagae T, Matsuoka $M$, et al. 2020. A common allosteric mechanism regulates homeostatic inactivation of auxin and gibberellin. Nat Commun 11(1): 2143.

Terol J, Domingo C, Talon M. 2006. The GH3 family in plants: genome wide analysis in rice and evolutionary history based on EST analysis. Gene 371(2): 279-290.

Thalmann M, Pazmino D, Seung D, Horrer D, Nigro A, Meier T, Kolling K, Pfeifhofer HW, Zeeman SC, Santelia D. 2016. Regulation of Leaf Starch Degradation by Abscisic Acid Is Important for Osmotic Stress Tolerance in Plants. Plant Cell 28(8): 1860-1878.

Uddin MN, Hossain MA, Burritt DJ. 2016. Salinity and drought stress: Similarities and differences in oxidative responses and cellular redox regulation. In Water Stress and Crop Plants: A Sustainable Approach; Ahmad, P., Ed.; John Wiley \& Sons: Singapore: 86-101.

Verslues PE, Agarwal M, Katiyar-Agarwal S, Zhu J, Zhu JK. 2006. Methods and concepts in quantifying resistance to drought, salt and freezing, abiotic stresses that affect plant water status. Plant J 45(4): 523-539.

Wang Y, Li K, Li X. 2009. Auxin redistribution modulates plastic development of root system architecture under salt stress in Arabidopsis thaliana. J Plant Physiol 166(15): 16371645.

Ware A, Walker CH, Simura J, Gonzalez-Suarez P, Ljung K, Bishopp A, Wilson ZA, Bennett T. 2020. Auxin export from proximal fruits drives arrest in temporally competent inflorescences. Nat Plants 6(6): 699-707.

Westfall CS, Sherp AM, Zubieta C, Alvarez S, Schraft E, Marcellin R, Ramirez L, Jez JM. 2016. Arabidopsis thaliana GH3.5 acyl acid amido synthetase mediates metabolic crosstalk in auxin and salicylic acid homeostasis. Proc Natl Acad Sci U S A 113(48): 13917-13922.

Wilson-Sanchez D, Rubio-Diaz S, Munoz-Viana R, Perez-Perez JM, Jover-Gil S, Ponce MR, Micol JL. 2014. Leaf phenomics: a systematic reverse genetic screen for Arabidopsis leaf mutants. Plant J 79(5): 878-891.

Yu Z, Duan X, Luo L, Dai S, Ding Z, Xia G. 2020. How Plant Hormones Mediate Salt Stress Responses. Trends Plant Sci 25(11): 1117-1130.

Zhang J, Lin JE, Harris C, Campos Mastrotti Pereira F, Wu F, Blakeslee JJ, Peer WA. 2016. DAO1 catalyzes temporal and tissue-specific oxidative inactivation of auxin in Arabidopsis thaliana. Proc Natl Acad Sci U S A 113(39): 11010-11015.

Zhang Q, Li J, Zhang W, Yan S, Wang R, Zhao J, Li Y, Qi Z, Sun Z, Zhu Z. 2012. The putative auxin efflux carrier OsPIN3t is involved in the drought stress response and drought tolerance. Plant J 72(5): 805-816. 
Zhang SW, Li CH, Cao J, Zhang YC, Zhang SQ, Xia YF, Sun DY, Sun Y. 2009. Altered architecture and enhanced drought tolerance in rice via the down-regulation of indole-3-acetic acid by TLD1/OsGH3.13 activation. Plant Physiol 151(4): 1889-1901.

Zhang Y, Li Y, Hassan MJ, Li Z, Peng Y. 2020. Indole-3-acetic acid improves drought tolerance of white clover via activating auxin, abscisic acid and jasmonic acid related genes and inhibiting senescence genes. BMC Plant Biol 20(1): 150.

Zhao C, Zhang H, Song C, Zhu J-K, Shabala S. 2020. Mechanisms of Plant Responses and Adaptation to Soil Salinity. The Innovation 1(1): 100017.

Zhao Y. 2018. Essential Roles of Local Auxin Biosynthesis in Plant Development and in Adaptation to Environmental Changes. Annu Rev Plant Biol 69: 417-435.

Zhao Z, Zhang Y, Liu X, Zhang X, Liu S, Yu X, Ren Y, Zheng X, Zhou K, Jiang L, et al. 2013. A role for a dioxygenase in auxin metabolism and reproductive development in rice. Dev Cell 27(1): 113-122.

Zheng Z, Guo Y, Novak O, Chen W, Ljung K, Noel JP, Chory J. 2016. Local auxin metabolism regulates environment-induced hypocotyl elongation. Nat Plants 2: 16025. 


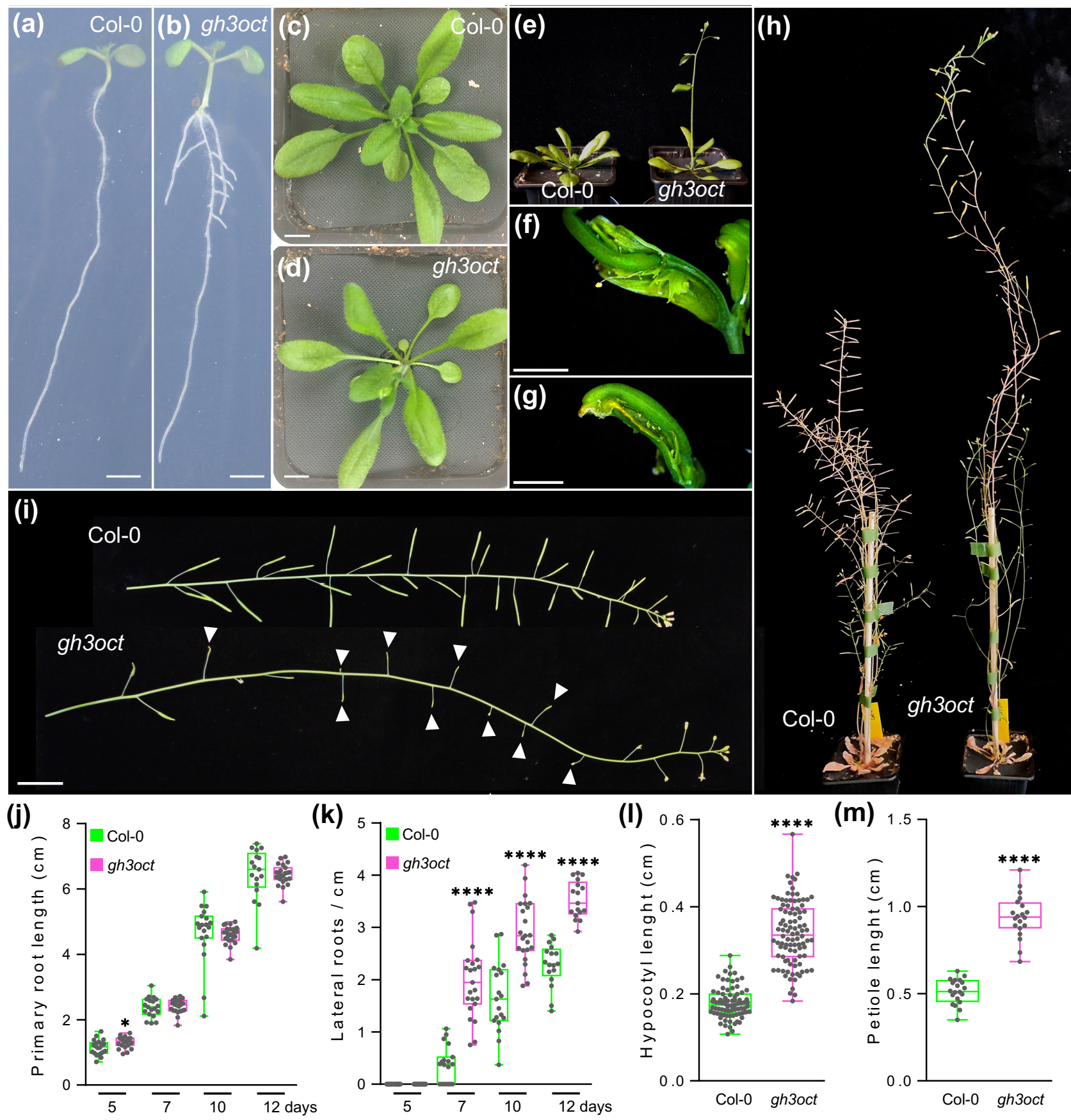


Casanova-Sáez et al. Figure 2

\section{- Col-0 gh3oct}
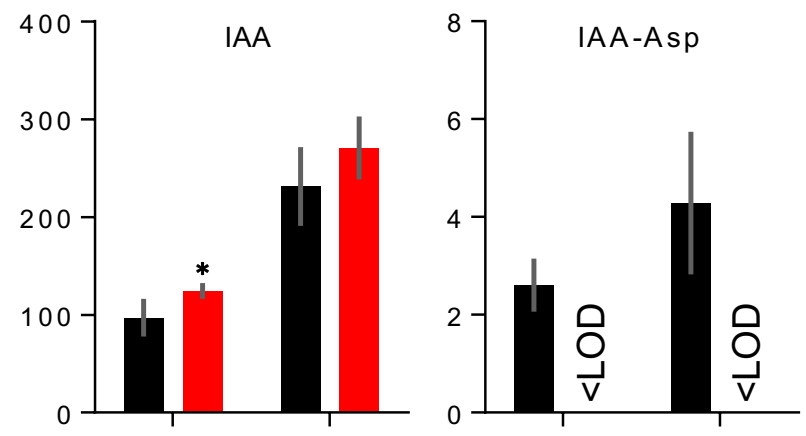

Shoot Roots

Shoot Roots

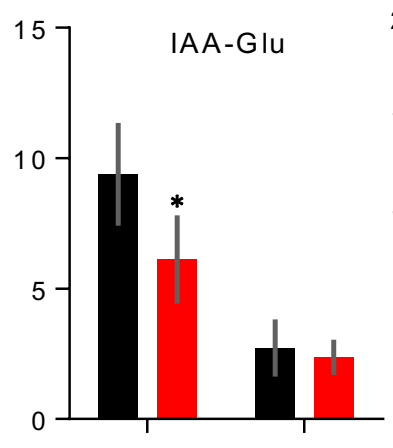

Shoot Roots

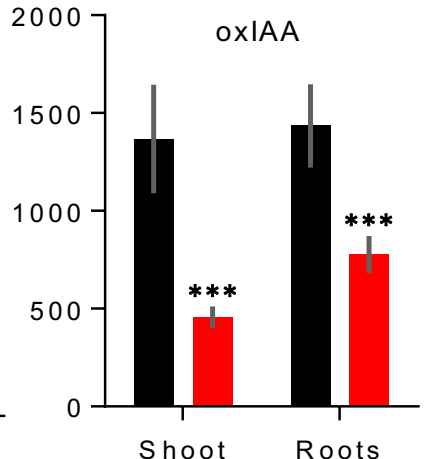

Shoot Roots

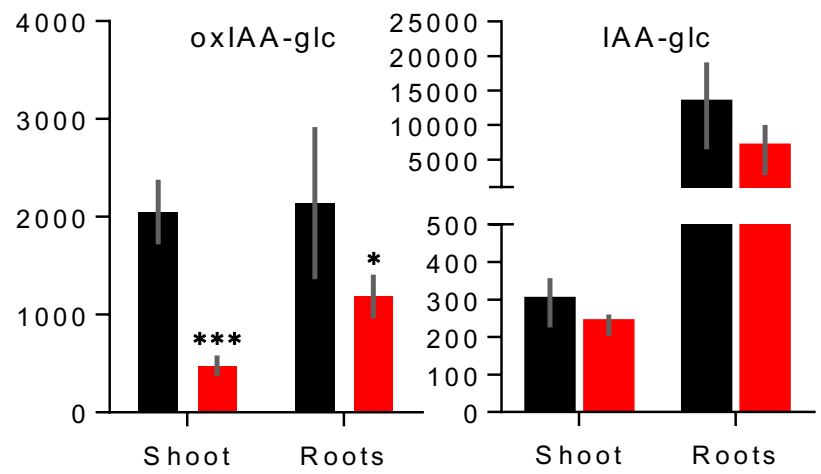


(a)

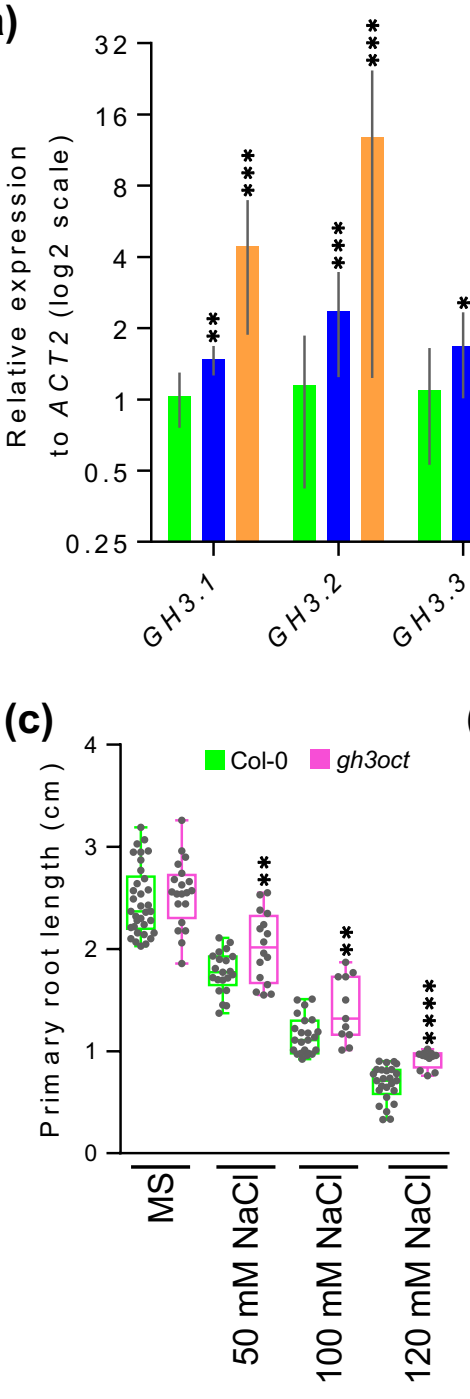

(b)

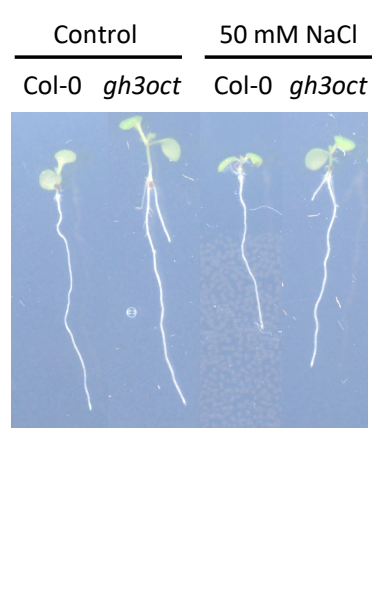

(e)

(d)

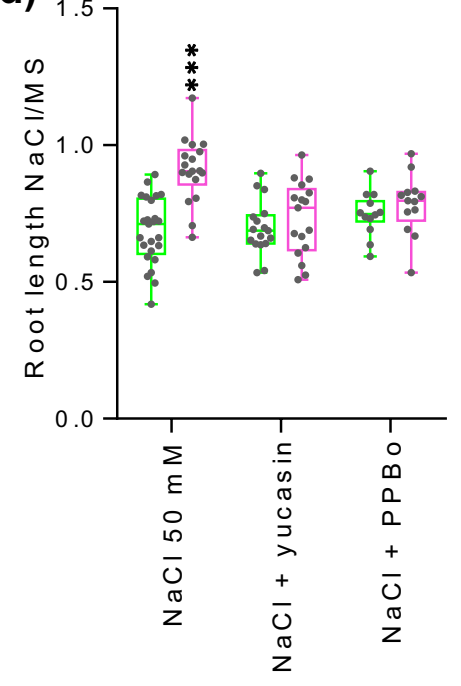

MS

$\mathrm{MS}+75 \mathrm{mM}$

$\mathrm{MS}+150 \mathrm{mM}$

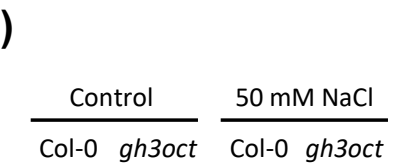


Casanova-Sáez et al. Figure 4

(a)

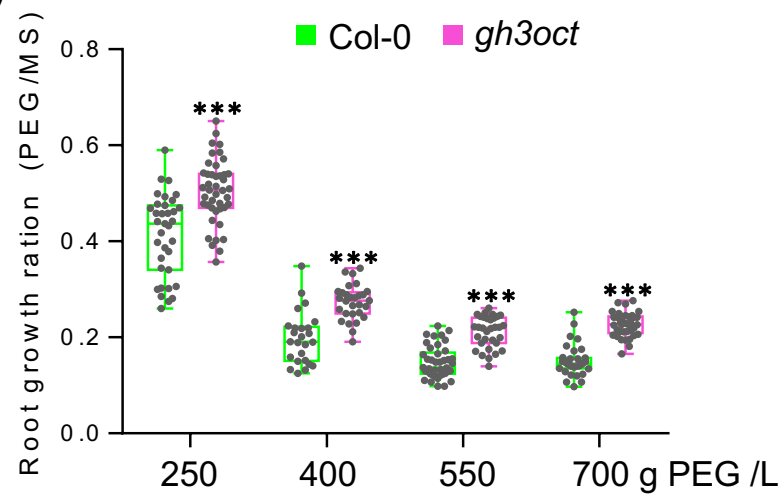

(b)

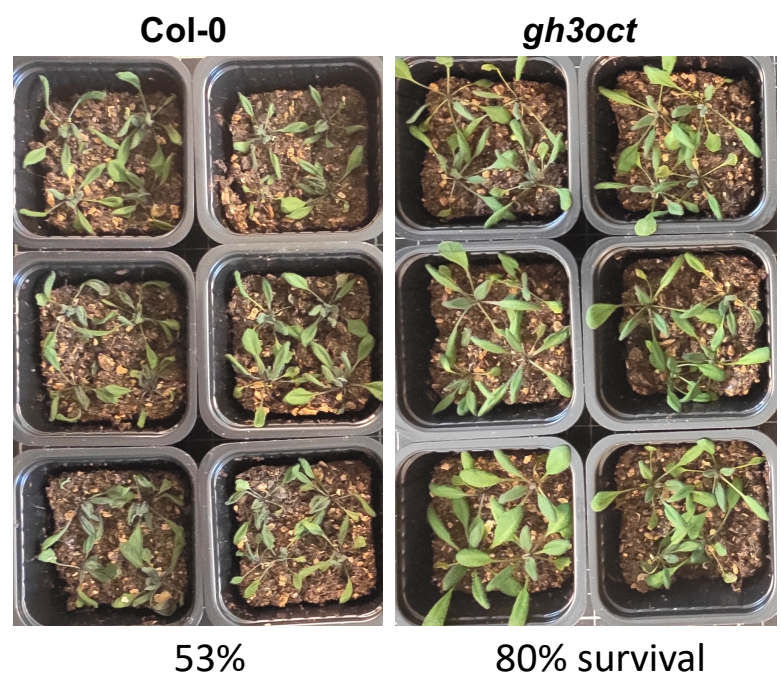

(c)

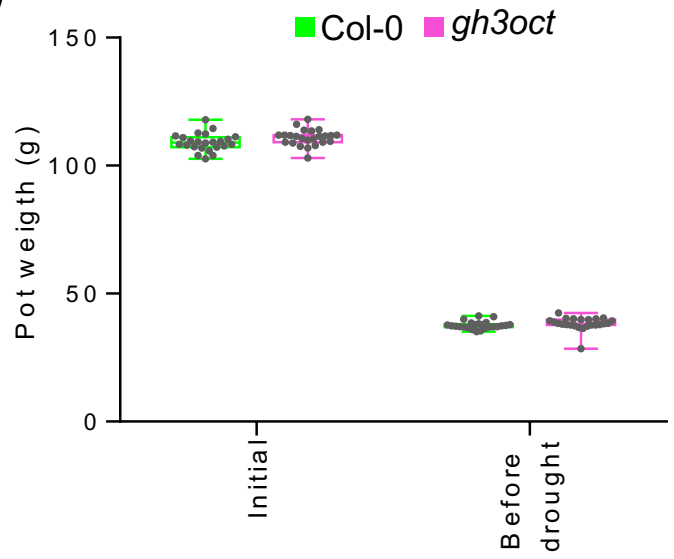


Casanova-Sáez et al. Figure 5
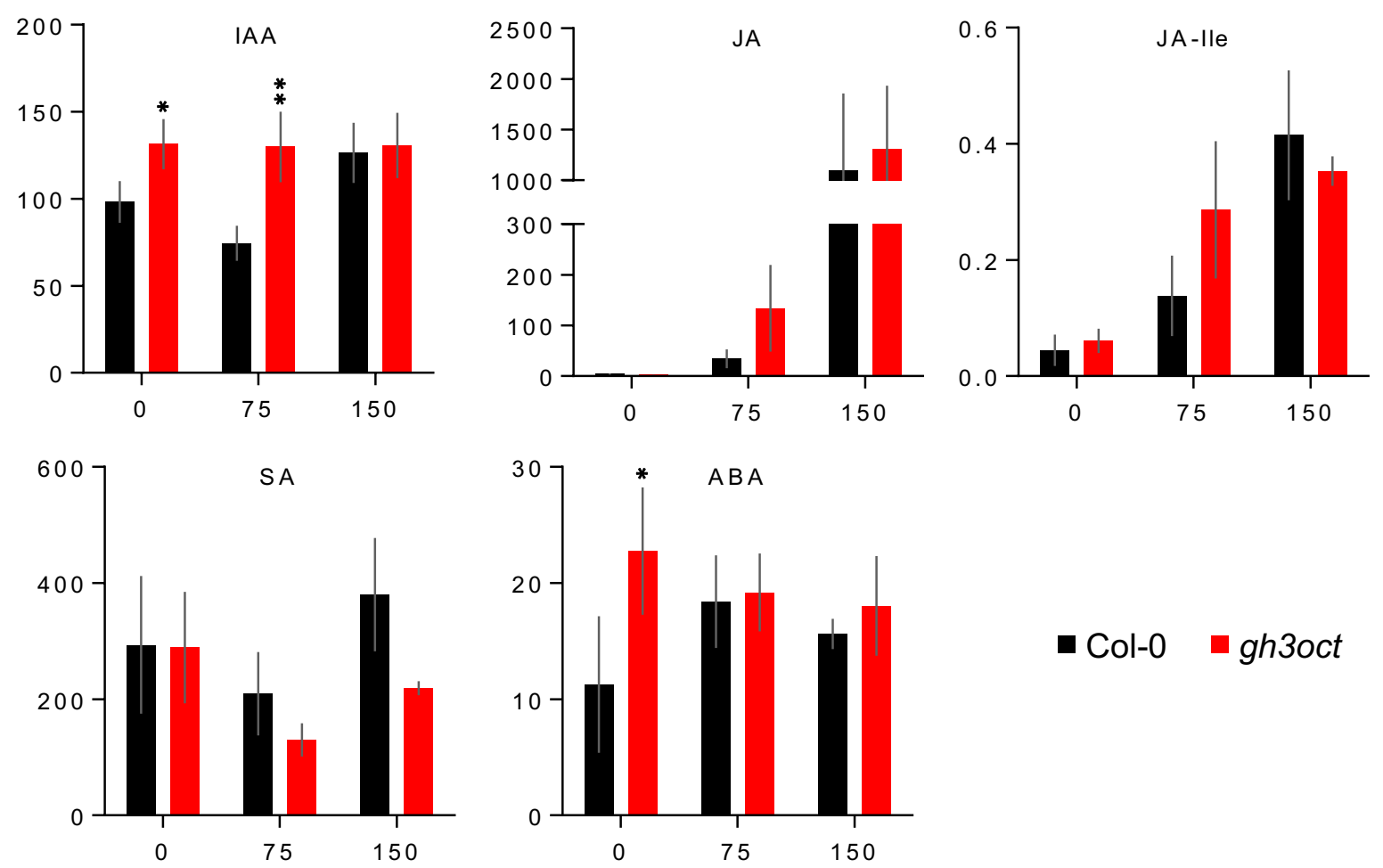

- Col-0 gh3oct 